\title{
Incremental Relaying with Imperfect Feedback in Wireless Cooperative Networks
}

\author{
Tobias Renk, Holger Jaekel, Friedrich. K. Jondral \\ Communications Engineering Lab \\ Karlsruhe Institute of Technology \\ Email: \{tobias.renk\}@kit.edu
}

\begin{abstract}
We present the $\epsilon$-outage capacity in the low signal-to-noise ratio (SNR) regime of an incremental relaying network. The network consists of one source, one destination, and two half-duplex relays. The one-bit feedback from the destination node is considered to be imperfect and is modeled as binary symmetric channel. The basic idea is that in each block only one terminal transmits depending on the feedback information in order to achieve a better use of the degrees of freedom of the channel. We derive a closed-form expression of the $\epsilon$-outage capacity and show that the quality of the feedback link has a great influence on the $\epsilon$-outage capacity of the network. We further examine an extension to the "usual" incremental relaying protocol by allowing the relays to overhear the transmissions of each other in order to increase their own decoding probability. This novel incremental relaying protocol performs close to the cut-set bound (within $0.5 \mathrm{bit} / \mathrm{s} / \mathrm{Hz}$ ).
\end{abstract}

Keywords - cooperative communications, incremental relaying, imperfect feedback, $\epsilon$-outage capacity

\section{INTRODUCTION}

The idea of incremental relaying was first introduced in [1]. The authors presented results for an amplify-and-forward relay network that operates in the high signal-to-noise ratio (SNR) regime. One of the main problems for the analysis of incremental relaying is the fact that the data rate itself is a random variable depending on the channel quality between the nodes. For that purpose, the notion of a long-term average data rate $\bar{R}$ was introduced in [1]. However, two problems occur with such a setting. First, there are several values of the initial target rate $R$ that lead to the same $\bar{R}$. The authors solved this issue by selecting the smallest possible $R$, i.e., that target rate that leads to the most reliable transmission. Second, consideration of the high SNR regime makes practically the use of incremental relaying obsolete. If the source is able to transmit with very high power, the probability that source-todestination transmission will fail tends to zero. Consequently, there is no need for the relay to transmit. The problem becomes much more realistic and interesting in the low SNR regime.

This was done for decode-and-forward in [2]. The authors derived the $\epsilon$-outage capacity for the general case of $K$ relays when there is perfect feedback from the destination. The $\epsilon$ outage capacity was then compared to that of the cut-set bound. For the one-relay case it has been shown that if the relay is located close to the source, incremental relaying with decode-and-forward is outage optimal in a way that it achieves the $\epsilon$-outage capacity of the cut-set bound. If the relay moves closer toward the destination, the gap between this protocol and the cut-set bound gets worse and worse. Furthermore, it was demonstrated that the optimal relay location only depends on the path-loss factor $\alpha$ and is independent of the SNR and the target outage probability $\epsilon$.

Variability of data rate dependent on the channel conditions is also treated in [3]. The authors examine a network in which the data rate is adapted with respect to the average SNR. The key point is to maintain a constant target outage probability at all SNR values. The main setting is a hybrid ARQ (automatic repeat request) protocol in Rayleigh block-fading channels.

Main Contributions: We derive the $\epsilon$-outage capacity of an incremental relaying protocol with two relays that employ decode-and-forward. One major distinction to prior work is that the feedback channel is considered to be imperfect. We stress that the maximal gain with respect to $\epsilon$-outage capacity compared to a network without feedback lies between 1 and 3 . This clearly points out the great advantages of feedback. Nevertheless, it must be highlighted that in practice feedback does not come at no costs. However, in our set-up we only consider a one-bit feedback that indicates success or failure of prior transmissions. Obviously, as the block length is large, the impact of one-bit on the data rate can be neglected and we get very good approximations of the true achievable rates. Furthermore, we propose an extension to the normal incremental relaying protocol that allows the relays to overhear the transmission of the other relay and, hence, increase their own decoding probability. This novel incremental relaying protocol performs close to the cut-set bound (within $0.5 \mathrm{bit} / \mathrm{s} / \mathrm{Hz}$ ).

The remainder of the paper is organized as follows. In Section II we introduce the system model. Section III deals with the derivation of the $\epsilon$-outage capacity of the proposed incremental relaying protocol. In Section IV we give numerical results and discuss our findings. Moreover, we introduce a novel incremental relaying scheme and analyze its $\epsilon$-outage capacity. Finally, Section V concludes the paper and gives some remarks on further research.

\section{System MODEL}

We consider the network depicted in Fig. 1 which consists of a source $S$, a destination $D$, and two relays $R_{1}$ and $R_{2}$, respectively. The channel gains $h_{i}, i \in\left\{\mathrm{sd}, \mathrm{sr}_{1}, \mathrm{sr}_{2}, \mathrm{r}_{1} \mathrm{~d}, \mathrm{r}_{2} \mathrm{~d}\right\}$, represent a slow Rayleigh fading profile with variances $\sigma_{i}^{2}$. As a consequence, $\left|h_{i}\right|^{2}$ follows an exponential distribution 


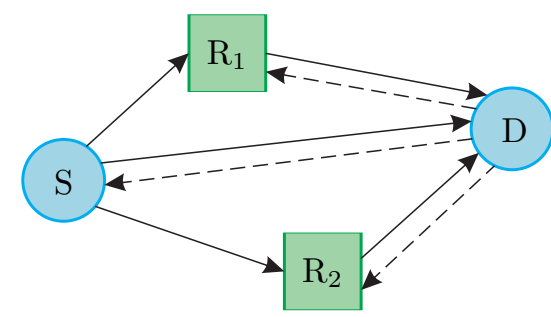

Fig. 1. Network for incremental relaying with imperfect feedback. Dashed lines indicate one-bit feedback from the destination to the source and the relays.

with mean value $\sigma_{i}^{2}$ and phases are uniformly distributed over $[0,2 \pi)$. We use a common path-loss model, where $\sigma_{i}^{2} \propto d_{i}^{-\alpha}$ with $d_{i}$ being the distance between two nodes. White Gaussian noise is added at each receiving node and noise realizations are assumed to be independent and identically distributed (i.i.d.). All noise realizations are drawn from $\mathcal{C N}(0, N)$. An average power constraint of $P$ is assumed at the source and the relays over a transmission block. The SNR is defined by $\mathrm{SNR}=P / N$. The relays are practically constrained in a way that they cannot transmit and receive at the same time (halfduplex relays). Furthermore, the relays employ decode-andforward, which means that they decode the source message and encode it again before retransmission. Throughout the paper it is assumed that there are enough channel uses per transmission phase so that the codes achieve their intended rates reliably if the rates are below the channel capacity.

The overall transmission block is divided into three subblocks. The transmission procedure then is as follows (see Fig. 2): During the first sub-block the source transmits to the relays and the destination. The destination then indicates success or failure of the source transmission by a one-bit feedback. If the source transmission was successful, the source allocates the next sub-block again. Since we assume block fading, it is obvious that this transmission will succeed as well. The same happens during the third sub-block and, hence, the overall gain becomes 3 in this case. If source transmission was not successful, then the relay $R_{1}$ transmits during the second sub-block. The destination now employs maximum ratio combining and tries to decode the combination of the source transmission and the relay transmission. Again, a onebit feedback indicates success or failure of decoding. If it was successful, the source occupies the third time slot and the whole procedure starts again. If it was not successful, relay $R_{2}$ transmits and the destination tries to decode the combination of all three transmission, i.e., the transmissions from the source, relay $R_{1}$, and relay $R_{2}$.

We use the $\epsilon$-outage capacity $\mathcal{C}_{\epsilon}$ as performance metric [4], which is defined as the highest rate $R$ such that outage probability satisfies $p_{\text {out }}(R, \mathrm{SNR}):=\operatorname{Pr}(\mathcal{C}(\mathrm{SNR})<R) \leq \epsilon$, where $0 \leq \epsilon \leq 1$ and $\mathcal{C}(\mathrm{SNR})$ is the instantaneous capacity. For a target outage probability $\epsilon$, we have $\mathcal{C}_{\epsilon}:=\sup \{R$ : $\left.p_{\text {out }}(R, \mathrm{SNR}) \leq \epsilon\right\}$.

Notation: $P_{\mathrm{SD}}$ describes the probability that the sourceto-destination transmission has been successful. Accordingly,

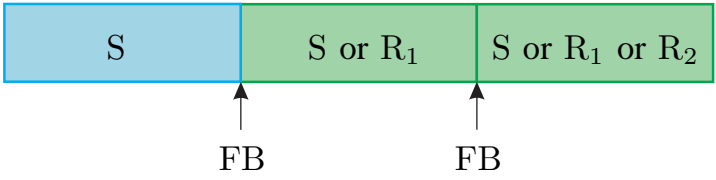

Fig. 2. Division of one transmission block into three sub-blocks. Source transmits during the first sub-block. Depending on the feedback (FB) either source of relay $R_{1}$ transmits during the second sub-block. Again, depending on the feedback, either source or relay $R_{1}$ or relay $R_{2}$ transmits.

$\bar{P}_{\mathrm{SD}}$ is the probability that the source-to-destination transmission has not been successful. $P_{\mathrm{R}_{1} \mathrm{D}}$, is the probability that the destination could decode after relay $R_{1}$ has transmitted. This also includes the preceding source transmission. Therefore, $P_{\mathrm{R}_{1} \mathrm{D}}$ means that the destination can decode after combining the transmissions from the source and the relay $\mathrm{R}_{1}$. Consequently, $\bar{P}_{\mathrm{R}_{1} \mathrm{D}}$ is the probability that the destination could not decode after the relay $R_{1}$ has transmitted. A positive acknowledgment from the destination is denoted by ACK and a negative acknowledgment by NACK. With $(\mathbf{A B})_{l \times m}$ we denote the element of the $l$-th row and the $m$-th column of the matrix product AB. The Hadamard (entry-wise) product of two matrices $\mathbf{A}$ and $\mathbf{B}$ is denoted as $\mathbf{A} \circ \mathbf{B}$. As we consider one-bit feedback, it is reasonable to model the feedback channels as binary symmetric channels (BSCs) defined as

$$
\begin{aligned}
p & :=\operatorname{Pr}(\mathrm{ACK} \mid \mathrm{ACK})=\operatorname{Pr}(\mathrm{NACK} \mid \mathrm{NACK}) \\
1-p & :=\operatorname{Pr}(\mathrm{NACK} \mid \mathrm{ACK})=\operatorname{Pr}(\mathrm{ACK} \mid \mathrm{NACK}) .
\end{aligned}
$$

For the sake of analysis, we assume that source and relays receive the feedback with the same degree of reliability, i.e., the feedback channel is the same and, hence, modeled by the same BSC with parameter $p$.

\section{OUTAGE CAPACITY}

\section{A. Baseline Setting}

We derive the $\epsilon$-outage capacity of incremental relaying by applying the method described in [2]. First, a baseline setting is examined, which means that we derive the $\epsilon$-outage capacity of the corresponding network but without feedback from the destination. After that, feedback comes into play by introducing a scaling factor that influences the pre-log factor [5]. The pre-log factor is similar to the multiplexing gain in the high SNR regime [6]. Each sub-block is assigned for a single transmission, i.e., we consider interference-free transmission (cf. Fig. 2). In order to have the same amount of information transmitted through the network (compared to direct transmission with rate $R$ ), each node transmits with $3 R$. For the sake of analysis, we define

$$
g(R, \mathrm{SNR}):=\frac{2^{3 R}-1}{\mathrm{SNR}}
$$

and drop the dependence on $R$ and SNR in the following.

An outage occurs for the following four cases:

1) The source signal cannot be decoded by the destination and by the relays. 
2) Relay $R_{1}$ can decode the source signal, but relay $R_{2}$ cannot. Additionally, the destination is still not able to decode after combining the signals from the source and relay $R_{1}$.

3) The same as 2), but the roles of the relays changed.

4) Both relays can decode, but the destination is still not able to decode after combining the signals from the source and both relays.

With these considerations, the outage probability of the network can be expressed as

$$
\begin{aligned}
& p_{\text {out }}=\operatorname{Pr}\left(\left|h_{\mathrm{sd}}\right|^{2}<g\right) \operatorname{Pr}\left(\left|h_{\mathrm{sr}_{1}}\right|^{2}<g\right) \operatorname{Pr}\left(\left|h_{\mathrm{sr}_{2}}\right|^{2}<g\right) \\
& +\operatorname{Pr}\left(\left|h_{\mathrm{sr}_{1}}\right|^{2} \geq g\right) \operatorname{Pr}\left(\left|h_{\mathrm{sr}_{2}}\right|^{2}<g\right) \operatorname{Pr}\left(\left|h_{\mathrm{sd}_{\mathrm{d}}}\right|^{2}+\left|h_{\mathrm{r}_{1} \mathrm{~d}}\right|^{2}<g\right) \\
& +\operatorname{Pr}\left(\left|h_{\mathrm{sr}_{1}}\right|^{2}<g\right) \operatorname{Pr}\left(\left|h_{\mathrm{sr}_{2}}\right|^{2} \geq g\right) \operatorname{Pr}\left(\left|h_{\mathrm{sd}}\right|^{2}+\left|h_{\mathrm{r}_{2} \mathrm{~d}}\right|^{2}<g\right) \\
& +\operatorname{Pr}\left(\left|h_{\mathrm{sr}_{1}}\right|^{2} \geq g\right) \operatorname{Pr}\left(\left|h_{\mathrm{sr}_{2}}\right|^{2} \geq g\right) \\
& \quad \operatorname{Pr}\left(\left|h_{\mathrm{sd}}\right|^{2}+\left|h_{\mathrm{r}_{1} \mathrm{~d}}\right|^{2}+\left|h_{\mathrm{r}_{2} \mathrm{~d}}\right|^{2}<g\right),
\end{aligned}
$$

where case 1) is represented by the first line on the right-hand side, case 2) by the second line, case 3 ) by the third line, and case 4) by the fourth and fifth line, respectively.

In order to be able to calculate the outage probability in the low SNR regime, we apply the following lemma (for the proof see [7]).

Lemma 1: Let $Y=\sum_{k=1}^{K} X_{k}$, where $X_{k}$ are independent exponentially distributed random variables with mean $\sigma_{k}^{2}$. If $g(\xi)$ is a continuous function at $\xi=0$ and $g(\xi) \rightarrow 0$ as $\xi \rightarrow 0$, then the cumulative distribution function $F_{Y}(\cdot)$ of $Y$ satisfies

$$
\lim _{\xi \rightarrow 0} \frac{F_{Y}(g(\xi))}{g(\xi)^{K}}=\frac{1}{K ! \prod_{k=1}^{K} \sigma_{k}^{2}} .
$$

Since we are interested in a low target error probability $\epsilon$, $\epsilon \rightarrow 0$ implies $g \rightarrow 0$. Therefore, if we examine the low SNR regime, i.e., SNR $\rightarrow 0$, we have to make sure that $2^{3 R}-1$ converges faster to zero than does SNR. Finally,

$$
\lim _{\substack { \epsilon \rightarrow 0 \\
\begin{subarray}{c}{\epsilon \rightarrow 0 \\
g \rightarrow 0{ \epsilon \rightarrow 0 \\
\begin{subarray} { c } { \epsilon \rightarrow 0 \\
g \rightarrow 0 } }\end{subarray}} \frac{p_{\text {out }}}{g^{3}}=\frac{6 \sigma_{\mathrm{r}_{1} \mathrm{~d}}^{2} \sigma_{\mathrm{r}_{2} \mathrm{~d}}^{2}+3 \sigma_{\mathrm{sr}_{1}}^{2} \sigma_{\mathrm{r}_{2} \mathrm{~d}}^{2}+3 \sigma_{\mathrm{sr}_{2}}^{2} \sigma_{\mathrm{r}_{1} \mathrm{~d}}^{2}+\sigma_{\mathrm{sr}_{1}}^{2} \sigma_{\mathrm{sr}_{2}}^{2}}{6 \sigma_{\mathrm{sd}^{2}}^{2} \sigma_{\mathrm{sr}_{1}}^{2} \sigma_{\mathrm{sr}_{2}}^{2} \sigma_{\mathrm{r}_{1} \mathrm{~d}}^{2} \sigma_{\mathrm{r}_{2} \mathrm{~d}}^{2}} .
$$

In the following, we substitute the right-hand side of (4) by $f(\boldsymbol{\sigma})^{-1}$. The $\epsilon$-outage capacity (without feedback) then becomes after some algebraic manipulations

$$
\mathcal{C}_{\epsilon}=\frac{1}{3} \log _{2}(1+\operatorname{SNR} \sqrt[3]{f(\boldsymbol{\sigma}) \epsilon})
$$

The $\epsilon$-outage capacity of the corresponding incremental relaying network is derived by multiplying $\mathcal{C}_{\epsilon}$ with the factor $3 / \mathbb{E}(N)$, where $\mathbb{E}(N)$ stands for the average amount of required transmission phases in order to send the source information to the destination. The reason for this scaling factor is the following. Assume the source transmission succeeds during the first sub-block. Then no relay has to transmit in the subsequent sub-blocks. Hence, we have $N=1$, where $N$ is the number of transmission phases. The next sub-block can then be occupied by the source and, due to the block fading model we consider, this transmission will be successful as well. The same is true for the third sub-block. To sum up, if the source transmission succeeds without any help by the relays, then the overall gain with respect to $\epsilon$-outage capacity becomes 3 . Now assume, in contrast, that the relay $R_{1}$ has to transmit, but not relay $R_{2}$. In this case, the third sub-block can be used by the source again to transmit the next information message and the gain over the baseline setting with respect to $\epsilon$-outage capacity becomes 1.5 . Similar consideration hold for other cases. Since the $\epsilon$-outage capacity is valid for all possible channel realizations and not only for instantaneous ones, the average of the transmission phases, i.e., $\mathbb{E}(N)$, must be taken into account.

\section{B. Number of Transmission Phases}

We now examine the required number of transmission phases (number of sub-blocks) in order to transmit a source message to the destination. Obviously, the maximum amount of required sub-blocks is 3 . In such a case the source and both relays would have transmitted to the destination. If the destination is still not able to decode, an outage is declared.

One sub-block is required for the following cases: 1) The source transmission was successful and ACK was received correctly. 2) The source transmission was not successful and NACK was received incorrectly.

Two sub-blocks are required if the following constellations occur: 1) The source transmission was successful and ACK was received incorrectly. Hence, the relay $R_{1}$ transmits. Then, we need two sub-blocks if the combination of the source and the relay transmissions was successful and ACK was received correctly or if the combined transmissions were not successful and NACK was received incorrectly. 2) The source transmission was not successful and NACK was received correctly. Again, the relay $R_{1}$ transmits and we get two sub-blocks if the combination of the source and the relay transmissions was successful and ACK was received correctly or if the combined transmissions were not successful and NACK was received incorrectly.

For the following four constellations, three sub-blocks are required:

1) The source-to-destination transmission succeeded, ACK was received incorrectly, combined transmissions of the source and the first relay succeeded, and ACK again was received incorrectly.

2) The source-to-destination transmission succeeded, ACK was received incorrectly, combined transmissions of the source and the first relay failed, and NACK was received correctly.

3) The source-to-destination transmission failed, NACK was received correctly, combined transmissions of the source and the first relay succeeded, and ACK was received incorrectly.

4) The source-to-destination transmission failed, NACK was received correctly, combined transmissions of the source and the first relay failed, and NACK again was received correctly. 
All these considerations can be summarized in the equation

$$
\begin{aligned}
\mathbb{E}(N)= & P_{\mathrm{SD}} p+\bar{P}_{\mathrm{SD}}(1-p) \\
& +2 P_{\mathrm{SD}}(1-p) P_{\mathrm{R}_{1} \mathrm{D}} p+2 P_{\mathrm{SD}}(1-p) \bar{P}_{\mathrm{R}_{1} \mathrm{D}}(1-p) \\
& +2 \bar{P}_{\mathrm{SD}} p P_{\mathrm{R}_{1} \mathrm{D}} p+2 \bar{P}_{\mathrm{SD}} p \bar{P}_{\mathrm{R}_{1} \mathrm{D}}(1-p) \\
& +3 P_{\mathrm{SD}}(1-p) P_{\mathrm{R}_{1} \mathrm{D}}(1-p)+3 P_{\mathrm{SD}}(1-p) \bar{P}_{\mathrm{R}_{1} \mathrm{D}} p \\
& +3 \bar{P}_{\mathrm{SD}} p P_{\mathrm{R}_{1} \mathrm{D}}(1-p)+3 \bar{P}_{\mathrm{SD}} p \bar{P}_{\mathrm{R}_{1} \mathrm{D}} p .
\end{aligned}
$$

It is obvious that, as the number of relays increases, derivation of $\mathbb{E}(N)$ becomes more and more complicated. Rearranging allows us to describe the average amount of required sub-blocks in a compact matrix notation. We get:

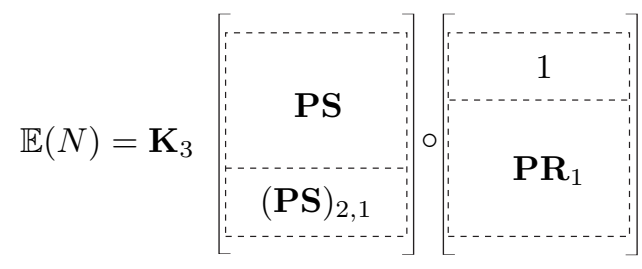

The vector $\mathbf{K}_{3}=[1,2,3]$ clearly is of dimension $(1 \times 3)$. The matrix $\mathbf{P}$ denotes the feedback channel and is given by

$$
\mathbf{P}=\left[\begin{array}{cc}
p & 1-p \\
1-p & p
\end{array}\right]
$$

The matrices $\mathbf{S}$ and $\mathbf{R}_{1}$ describe the decoding probability at the destination after the source transmission and after the combination of the source and the transmission of the relay $\mathrm{R}_{1}$, respectively. Accordingly, $\mathbf{S}=\left[P_{\mathrm{SD}}, \bar{P}_{\mathrm{SD}}\right]^{T}$ and $\mathbf{R}_{1}=\left[P_{\mathrm{R}_{1} \mathrm{D}}, \bar{P}_{\mathrm{R}_{1} \mathrm{D}}\right]^{T}$. Finally, $(\mathbf{P S})_{2,1}=P_{\mathrm{SD}}(1-p)+\bar{P}_{\mathrm{SD}} p$.

The average amount of required sub-blocks $\mathbb{E}(N)$ vs. the reliability $p$ of the feedback is shown in Fig. 3. The array of curves is bounded by a rectangular box given by $\mathbb{E}(N)=1$, $\mathbb{E}(N)=3, p=0$, and $p=1$. The shapes of the curves depend on the parameter set $\left\{P_{\mathrm{SD}}, P_{\mathrm{R}_{1} \mathrm{D}}\right\}$. The values used in Fig. 3 are listed in Tab. I. The gray area illustrates the region where no curves can be found. An interesting fact is that all curves intercept for $p=0.5$ (unreliable feedback). The average amount of required sub-blocks for this value is $\mathbb{E}(N)=1.75$.

TABLE I

VALUES FOR $P_{\mathrm{SD}}$ AND $P_{\mathrm{R}_{1} \mathrm{D}}$ IN FIG. 3.

\begin{tabular}{c||c|c|c|c|c} 
tag & $a$ & $b$ & $c$ & $d$ & $e$ \\
\hline \hline$P_{\mathrm{SD}}$ & 1 & 0.9 & 0.8 & 0.5 & 0 \\
\hline$P_{\mathrm{R}_{1} \mathrm{D}}$ & 1 & 0.95 & 0.85 & 0.5 & 0
\end{tabular}

\section{NumericAl Results AND Discussion}

In this section we give numerical results for the investigated protocols and discuss our findings. For simulations we use a one-dimensional geometry as shown in Fig. 4.

The upper bound (i.e., $\mathbb{E}(N)=1$ ) for the $\epsilon$-outage capacity of incremental relaying is illustrated in Fig. 5. The $\epsilon$-outage capacities of the baseline setting and the general upper bound (cut-set bound) are also depicted. A target outage probability

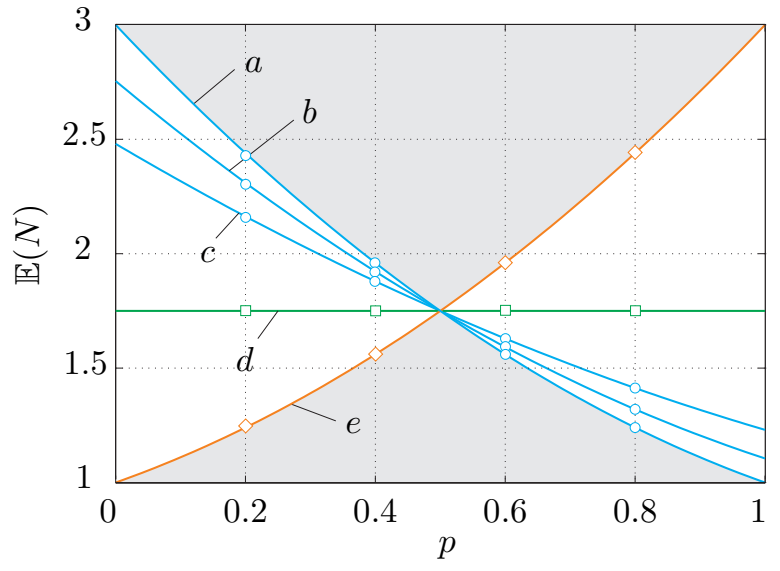

Fig. 3. Average amount of required sub-blocks $\mathbb{E}(N)$ vs. reliability $p$ of the feedback for the two-relay case.

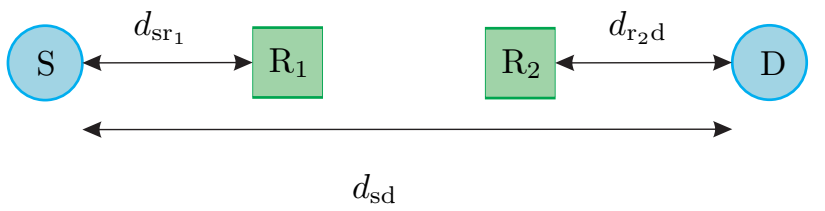

Fig. 4. One-dimensional geometry used for the analysis. The distances have been normalized to the source-to-destination distance, i.e., $d_{\mathrm{sd}}=1, d_{\mathrm{sr}_{1}}=$ $1 / 3$, and $d_{\mathrm{r}_{2} \mathrm{~d}}=1 / 3$, respectively. Note that $d_{\mathrm{r}_{1} \mathrm{~d}}=1-d_{\mathrm{sr}_{1}}=2 / 3$ and $d_{\mathrm{sr}_{2}}=1-d_{\mathrm{r}_{2} \mathrm{~d}}=2 / 3$.

of $\epsilon=10^{-3}$ was used and the path-loss factor was set to $\alpha=4$. We see that with incremental relaying we achieve enormous gains over the simple baseline setting where each relay transmits in its assigned sub-block even if source transmission was successful. Nevertheless, the gap between incremental relaying and the cut-set bound is remarkable. For instance, consider an SNR of $5 \mathrm{~dB}$. The difference between the cut-set bound and incremental relaying is approximately $1 \mathrm{bit} / \mathrm{s} / \mathrm{Hz}$. The reason for this is obvious: Decoding at relay $R_{2}$ is only based on the source transmission. However, the transmission of relay $R_{1}$ during the second sub-block can be exploited in order to increase the decoding probability of $\mathrm{R}_{2}$. If we do so, we get an $\epsilon$-outage capacity given by

$$
\mathcal{C}_{\epsilon}^{\left(\mathrm{IR}^{\prime}\right)}=\frac{1}{\mathbb{E}(N)} \log _{2}(1+\operatorname{SNR} \sqrt[3]{\tilde{f}(\boldsymbol{\sigma}) \epsilon})
$$

with $\tilde{f}(\boldsymbol{\sigma})=\sigma_{\mathrm{sd}^{2}}^{2} \sigma_{\mathrm{sr}_{1}}^{2} \sigma_{\mathrm{sr}_{2}}^{2}$. (The derivation of the above equation is given in the Appendix.) An upper bound (i.e., $\mathbb{E}(N)=1$ ) of this protocol is shown in Fig. 5, too. It can easily be seen that the gap between the cut-set bound and the novel incremental relaying protocol for $\mathrm{SNR}=5 \mathrm{~dB}$ is only $0.3 \mathrm{bit} / \mathrm{s} / \mathrm{Hz}$. Asymptotically, the maximal gap becomes $0.5 \mathrm{bit} / \mathrm{s} / \mathrm{Hz}$. The major advantage of this protocol is that the gain comes at almost no cost, because we simply exploit the broadcast nature of the wireless channel. 


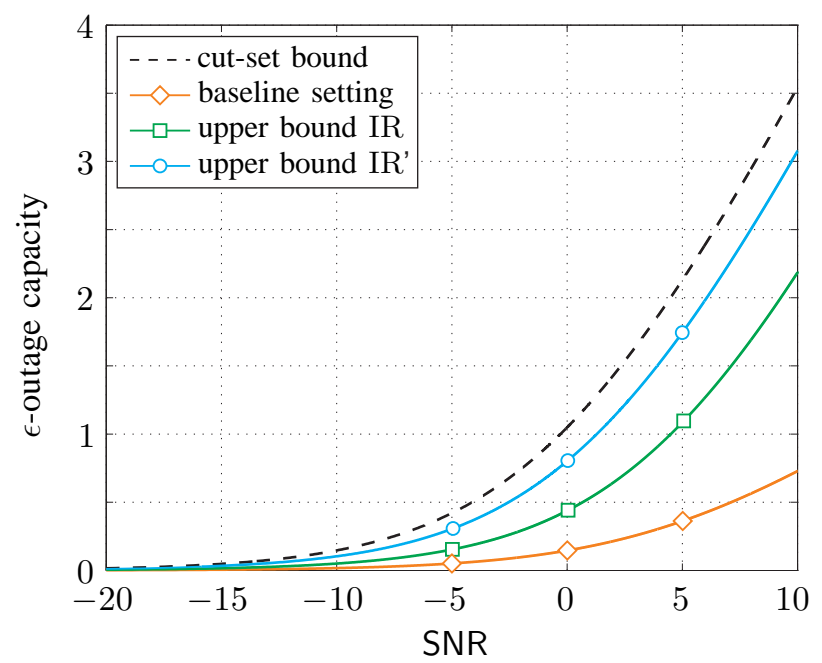

Fig. 5. $\epsilon$-outage capacity in bit/s/Hz vs. SNR in $\mathrm{dB}$ for $\epsilon=10^{-3}, \alpha=4$, and the geometrical constellation shown in Fig. 4.

\section{Conclusions}

We investigated the $\epsilon$-outage capacity of an incremental relaying network in the low SNR regime. The network consists of one source node, one destination node, and a pair of halfduplex relays. It has been demonstrated that incremental relaying achieves great performance gains over a simple decodeand-forward relaying protocol. Nevertheless, the performance gap between the cut-set bound and incremental relaying is remarkable. In order to overcome this drawback, we extended the normal incremental relaying protocol by allowing the relays to overhear the transmission of the other relay. In doing so, each relay increases its own ability to decode and, thus, increases the overall $\epsilon$-outage capacity enormously. It has been shown that this novel incremental relaying protocol performs close to the cut-set bound (within $0.5 \mathrm{bit} / \mathrm{s} / \mathrm{Hz}$ ). We currently deal with the extension of this protocol to large networks with an arbitrary number of relays. Moreover, comparison to the incremental relaying network with bursty amplify-and-forward as proposed in [8] is under investigation.

\section{APPENDIX}

The derivation of the $\epsilon$-outage capacity of the novel incremental relaying protocol is similar to the one described in Subsection III-A. We first derive the $\epsilon$-outage capacity of the corresponding baseline setting (in our case this means that relay $R_{2}$ receives the transmission from relay $R_{1}$ as well) and then multiply the result with $3 / \mathbb{E}(N)$. An outage occurs for the following cases.

1) Neither the relays nor the destination can decode the source signal.

2) $R_{1}$ can decode the source signal, but the destination and $R_{2}$ cannot. Then, the destination and $R_{2}$ still cannot decode the combined signals from the source and $R_{1}$.

3) $R_{1}$ can decode the source signal, but the destination and $\mathrm{R}_{2}$ cannot. After the second sub-block, the destination still cannot decode, but $\mathrm{R}_{2}$ can. Then, the destination still cannot decode the combined signal from the source, $\mathrm{R}_{1}$, and $\mathrm{R}_{2}$.

4) Similar to 2) and 3), but the roles of $R_{1}$ and $R_{2}$ changed.

5) The destination cannot decode the source signal after the first sub-block, but both relays can. After the second sub-block, the destination still cannot decode. Even after the third sub-block, the destination is still not able to decode.

We now apply Lemma 1 for $K=3$. Thus, the only term that is nonzero is the one described in 1). All other terms tend to zero for the limit $g \rightarrow 0$. We demonstrate this by showing two examples. First, consider 1). We get:

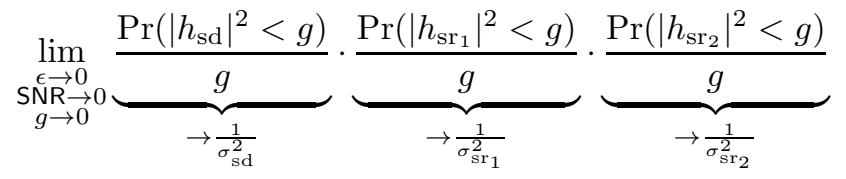

Now, consider 2). We have:

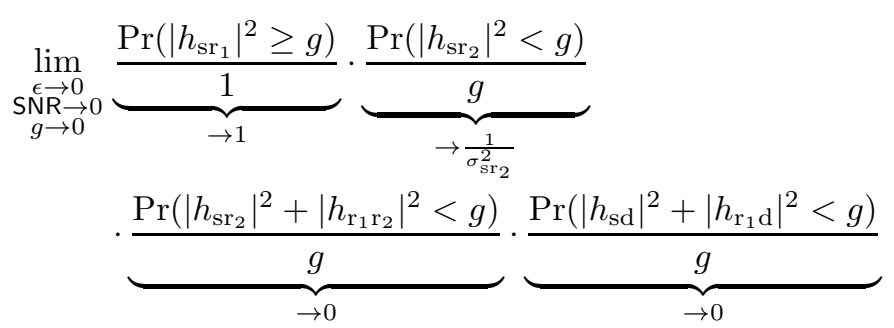

Hence, we finally get

$$
\lim _{\substack{\epsilon \rightarrow 0 \\ \text { SNR } \rightarrow 0 \\ g \rightarrow 0}} \frac{p_{\text {out }}}{g^{3}}=\frac{1}{\sigma_{\text {sd }^{2}} \sigma_{\mathrm{sr}_{1}}^{2} \sigma_{\mathrm{sr}_{2}}^{2}},
$$

which yields after some algebraic manipulation the desired result for $\mathcal{C}_{\epsilon}^{\left(\mathrm{IR}^{\prime}\right)}$.

\section{REFERENCES}

[1] J. Laneman, D. Tse, and G. Wornell, "Cooperative diversity in wireless networks: Efficient protocols and outage behavior," IEEE Transactions on Information Theory, vol. 50, no. 12, pp. 3062-3080, December 2004.

[2] T. Renk, H. Jaekel, F. Jondral, D. Gündüz, and A. Goldsmith, "Outage capacity of incremental relaying at low signal-to-noise ratios," IEEE 70th Vehicular Technology Conference (VTC-Fall), Anchorage, Alaska, September 2009.

[3] P. Wu and N. Jindal, "Performance of hybrid-arq in blockfading channels: A fixed outage probability analysis," available at: http://arxiv.org/pdf/0811.4191, November 2008.

[4] L. H. Ozarow, S. Shamai (Shitz), and A. D. Wyner, "Information theoretic considerations for cellular mobile radio," IEEE Transactions on Vehicular Technology, vol. 43, no. 2, pp. 359-377, May 1994.

[5] B. Rankov and A. Wittneben, "Spectral efficient signaling for halfduplex relay channels," Conference Record of the Thirty-Ninth Asilomar Conference on Signals, Systems and Computers, pp. 1066-1071, 2005.

[6] L. Zheng and D. Tse, "Diversity and multiplexing: A fundamental tradeoff in multiple antenna channels," IEEE Transactions on Information Theory, vol. 49, pp. 1073-1096, May 2003.

[7] A. Avestimehr and D. Tse, "Outage capacity of the fading relay channel in the low-snr regime," IEEE Transactions on Information Theory, vol. 53, no. 4, pp. 1401-1415, April 2007.

[8] T. Renk, H. Jaekel, F. Jondral, D. Gündüz, and A. Goldsmith, "Outage capacity of bursty amplify-and-forward with incremental relaying," submitted to International Symposium on Information Theory, Austin, Texas, USA, June 2010. 